\title{
To Eat or Not to Eat. The Good and Bad of Eating Barley in Type 2 Diabetic Patients
}

\author{
Anab Fatima* \\ Dow College of Pharmacy, Dow University of Health Sciences, Karachi, Pakistan.
}

\begin{abstract}
In the previous several decades it has been noted that prevalence rate of Type 2 diabetes dramatically rising worldwide. The substantial increase in morbidity and mortality associated to type 2 diabetes along with the overhead cost for management of the disease render it onerous global health problem. This scenario required to do serious intervention to overcome its complications. The author focused on management of diabetes mellitus through intake of barley in the diet to highlight it's importance whether it has any long term effect on the management and control of diabetes mellitus.
\end{abstract}

Keywords: Barley, Hordeum vulgare L, Diabetes mellitus, Complications, Hyperglycemia.

\section{INTRODUCTION}

Hyperglycemia and improper secretion of insulin are most evident in Diabetes Mellitus (DM) which is one of the most important metabolic disorders. When it becomes chronic it leads to ketosis and proteinuria, and further proceeds to a number of complications like retinopathy, nephropathy, and neuropathy. In more severe stage it develop peripheral vascular insufficiencies that can lead to limb amputation [1]. This require an urgent and focused interventions by the stakeholders not only to prevent or extend the development of type 2 diabetes but also to combat its complications. Among all these interventions diet has more significant importance. Nevertheless it requires more understanding that which dietary element significantly reduce the prevalence and cause the regression of type 2 diabetes. As narrated by Guoyong Yu and colleagues [2] in their article that diet containing bioactive compounds may be beneficial for human health. Tomoe Watanabe and the colleagues [3] further highlighted the recommendations of Canadian Diabetes Association (CDA). They advised for consumption of food with low glycemic index in consultation with registered dietitian which is to be followed by regular monitoring to encourage dietary adherence. Jamilah M. Hashemi [4] reviewed various studies particularly for diet containing whole grain such as Hordeum vulgare $L$. (HV or barley) and chance of occurrence of different diseases. She gave evidences that long term consumption of barley plays a major role for the control of diabetes due to high fiber contents. It reduces glycemia, insulinemia and lipemia in Type 2 diabetes.

Further, in their article Saima Gul [5] and colleagues proposed possible mechanisms for anti-inflammatory and cardiovascular effects of $\mathrm{HV}$ in traditional medicine.

*Address correspondence to this author at the Dow College of Pharmacy, Dow University of Health Sciences, Karachi, Pakistan. E-mail: anabfatima@gmail.com
In response of all that work the authors want to mention here matter of great concern. It has been clear from previous studies that barley is beneficial for chronic diseases but their still remain work to done for its incorporation as an effective medication for treatment of chronic disease. The concept of anti-inflammatory, thrombolytic and cardiovascular effects proposed by Saima Gul [5] and colleagues require to further explore the mechanism. Although it is real fact that barley may be a useful dietary approach to lowering the risk of developing diabetes but the exact mechanism is still unclear and require lots of work to prove it. As suggested by Moonyeon Youn and colleagues [6] that in the early stages of type 2 diabetes consumption of whole grains may offer modest benefit in rats, but this benefit is lost with further progression of the disease. The bioavailability of bioactive component is debatable and need further evaluation and clinical investigations in order to use them as a novel compound for the treatment and preventive cure of chronic disease. Furthermore, effect of consuming barley merit more detailed study. In this context fractionation and isolation of pure compound could provide valuable knowledge and it could be matter of interest for the researcher.

\section{CONFLICT OF INTEREST}

Declared none.

\section{ACKNOWLEDGEMENTS}

Declared none.

\section{REFERENCES}

[1] Minaiyan M, Ghannadi A, Movahedian A, Hakim-Elahi I. Effect of Hordeum vulgare L. (Barley) on blood glucose levels of normal and STZ-induced diabetic rats. Res Pharm Sci 2014; 9(3): 173-8. 
[2] Yu G, Wang F, Zhang B, Fan J. In vitro inhibition of platelet aggregation by peptides derived from oat (Avena sativa L.), highland barley (Hordeum vulgare Linn. var. nudum Hook. f.), and buckwheat (Fagopyrum esculentum Moench) proteins. Food Chem 2016; 194: 577-86. DOI: 10.1016/j.foodchem.2015.08.058

[3] Watanabe T, Berry TR, Willows ND, Bell RC. Assessing intentions to eat low-glycemic index foods by adults with diabetes using a new questionnaire based on the theory of planned behaviour. Can J Diabetes 2015; 39: 94-100. DOI: 10.1016/j.jcjd.2014.09.001
[4] Hashemi JM. Biomedical effects of barley- a review. NY Sci J 2015; 8(3): 52-5.

[5] Gul M, Ahmed S, Kifli S, et al. Multiple pathways are responsible for anti-inflammatory and cardiovascular activities of Hordeum vulgare L. J Transl Med 2014; 12: 316. DOI: 10.1186/s12967-014-0316-9

[6] Youn M, Csallany AS, Gallaher DD. Whole grain consumption has a modest effect on the development of diabetes in the Goto-Kakisaki rat. Br J Nutr 2012; 107: 192-201. DOI: $10.1017 /$ S0007114511002741

(C) 2017 National journal of health sciences.

This is an open-access article. 